\title{
Novel oral contraceptive for heavy menstrual bleeding: estradiol valerate and dienogest
}

REVIEW

This article was published in the following Dove Press journal:

International Journal of Women's Health

II June 2013

Number of times this article has been viewed

\author{
Sally Rafie' \\ Laura Borgelt ${ }^{2}$ \\ Erin R Koepf ${ }^{3}$ \\ Mary E Temple-Cooper ${ }^{4}$ \\ K Joy Lehman ${ }^{5}$ \\ 'Department of Pharmacy, University \\ of California San Diego Health \\ System, San Diego, CA, ${ }^{2}$ Departments \\ of Clinical Pharmacy and Family \\ Medicine, University of Colorado \\ Anschutz Medical Campus, Aurora, \\ CO, ${ }^{3}$ Department of Pharmacy \\ Practice, University of New England \\ College of Pharmacy, Portland, ME, \\ ${ }^{4}$ Department of Pharmacy, Hillcrest \\ Hospital, Cleveland Clinic, Mayfield \\ Heights, $\mathrm{OH},{ }^{5}$ Department of \\ Pharmacy, Ohio State University \\ Medical Center, Columbus, $\mathrm{OH}$, USA
}

Correspondence: Sally Rafie UC San Diego Health System, 200 West Arbor Dr \#8765,

San Diego, CA 92103 , USA

$\mathrm{Tel}+|\mathrm{l} 619543360|$

Fax +I 6195435829

Email srafie@ucsd.edu
Abstract: Abnormal uterine bleeding (AUB) is associated with significant direct medical costs and impacts both society and the quality of life for individual women. Heavy menstrual bleeding, a subset of AUB, also referred to as menorrhagia, is defined as menstrual blood loss greater than $80 \mathrm{~mL}$ or the patient's perception of excessive blood loss. The newest treatment option available is a novel combination oral contraceptive product containing estradiol valerate (E2V) and dienogest (DNG). As with other combination oral contraceptives, E2V/DNG works primarily by preventing ovulation. However, in contrast with other combination oral contraceptives, it is the progestin component of E2V/DNG that is responsible for endometrial stabilization. Use of E2V/DNG for six months has led to significant reductions in heavy menstrual bleeding with an average $65 \%$ reduction in mean blood loss. Approximately half of the women with heavy menstrual bleeding who received E2V/DNG for six months demonstrated an $80 \%$ reduction in mean blood loss. Additionally, significant improvements in hematologic indicators (ie, ferritin, hemoglobin, and hematocrit) have been shown. Based on its chemical properties, E2V/DNG may have fewer adverse effects on lipid and glucose metabolism and reduced risk of thromboembolic complications compared with other combination oral contraceptives. This has not yet been shown in clinical trials and until then it should be assumed that E2V/DNG has a safety profile similar to other combination oral contraceptives containing $35 \mu \mathrm{g}$ or less of ethinyl estradiol. E2V/DNG has been compared with another combination oral contraceptive in healthy women without heavy menstrual bleeding and demonstrated improved bleeding patterns. E2V/DNG has not been compared with the levonorgestrel intrauterine device or other treatments for heavy menstrual bleeding. When compared with some other treatment options for AUB, E2V/DNG provides the added advantage of effective contraception.

Keywords: contraception, dienogest, estradiol valerate, Natazia ${ }^{\circledR}$, menstrual disorders, menorrhagia

\section{Introduction}

Abnormal uterine bleeding (AUB) is a prevalent complaint among women of reproductive age. ${ }^{1}$ Nearly one-third of outpatient gynecologist visits and over 400,000 hospitalizations a year can be attributed to AUB. ${ }^{2,3}$ This is associated with significant direct medical costs and impacts both society and quality of life for individual women, due to loss of work productivity and limitations to leisure activity. ${ }^{4-6}$ In the US alone, AUB has direct costs of \$1 billion and indirect costs of \$12 billion according to conservative estimates. ${ }^{7}$ Women with AUB have a health-related quality of life below the 25 th percentile of that for the general population of women within a similar age range. ${ }^{7}$ 
AUB in women of reproductive age is characterized by menstrual flow outside of normal volume, duration, regularity, or frequency and can present anytime from adolescence to menopause. ${ }^{8}$ The diagnosis can be further classified as heavy menstrual bleeding or intermenstrual bleeding. ${ }^{6,8}$ Heavy menstrual bleeding, also referred to as menorrhagia, is defined as menstrual blood loss greater than $80 \mathrm{~mL}$ or the patient's perception of excessive blood loss. ${ }^{8,9}$

Given that up to $20 \%$ of patients presenting with heavy menstrual bleeding have a bleeding disorder, all patients should be screened for an underlying bleeding disorder with a thorough medical history, and if indicated, further hematologic testing. ${ }^{10-13}$ Medications such as anticoagulants and nonsteroidal anti-inflammatory drugs (NSAIDs) or herbal remedies, such as ginkgo biloba and ginseng, should also be considered as contributors to heavy menstrual bleeding. Other structural etiologies, including polyps, adenomyosis, leiomyoma, and hyperplasia/malignancy, and nonstructural etiologies, including anovulation and polycystic ovary syndrome, should be considered in the differential diagnosis.

Following appropriate gynecologic evaluation, medical, radiological (ie, uterine artery embolization), or surgical management (ie, hysterectomy) may be indicated. While hysterectomy cures heavy menstrual bleeding, it is associated with operative risk and postoperative morbidity that can be avoided with less invasive treatments when appropriate. Many etiologies of heavy menstrual bleeding can be adequately managed with uterine-preserving pharmacotherapy. Therapies include NSAIDs, antifibrinolytics (ie, tranexamic acid), or hormonal modulators (ie, danazol), including oral progestins, combination estrogen/progestin oral contraceptives, gonadotropin receptor antagonists, and the levonorgestrel-releasing intrauterine device (LNG-IUD).

The newest treatment option available is a novel combination estrogen/progestin oral contraceptive product containing estradiol valerate (E2V) and dienogest (DNG). This quadriphasic product was developed to improve upon prior E2V oral contraceptive product weaknesses. The product received US Food and Drug Administration approval for prevention of pregnancy in 2010 and for the treatment of heavy menstrual bleeding in 2012. This article will review the pharmacology and mechanism of action of E2V/DNG, as well as its efficacy in treating heavy menstrual bleeding and place in therapy.

\section{Literature review}

In order to review the mechanism of action, pharmacology, pharmacokinetics, efficacy, safety, and place in therapy of
E2V/DNG for heavy menstrual bleeding, a PubMed search restricted to English language articles citing estradiol valerate or dienogest from 1985 to January 2013 was conducted. Additional data sources were identified from the references of selected articles. Available data sources were then evaluated to summarize the mechanism of action, pharmacology, pharmacokinetics, efficacy, safety, and place in therapy of E2V/DNG when used for heavy menstrual bleeding.

\section{Mechanism of action, pharmacology, and pharmacokinetics}

As with other combination oral contraceptives, E2V/DNG works primarily by preventing ovulation. ${ }^{14}$ Combination oral contraceptives provide negative feedback to the hypothalamicpituitary-ovarian axis. This suppresses ovulation by lowering gonadotropin-releasing hormone pulsation and the pituitary response to gonadotropin-releasing hormone. The estrogen component prevents the release of follicle-stimulating hormone from the pituitary and in turn prevents follicle development. The progestin component prevents the surge of luteinizing hormone from the pituitary gland and subsequent release of the dominant follicle. DNG also increases cervical mucosa thickness, resulting in decreased sperm motility, and prevents proliferation of the endometrium. These additional effects may prevent implantation.

In most combination oral contraceptives, the estrogen component is responsible for providing cycle control and stabilizing the endometrium for an acceptable bleeding pattern. E2V, unlike ethinyl estradiol, did not provide an adequate level of endometrial stability, as evidenced by breakthrough bleeding rates in preliminary studies. ${ }^{15}$ For this reason, E2 V-containing combination oral contraceptives did not reach the marketplace until this novel combination of E2V and DNG was evaluated. ${ }^{15}$

The effects of a $2 \mathrm{mg} /$ day dose of E2V on the hypothalamic-pituitary-ovarian axis and impact on endometrium and ovarian function are expected to be similar to that of a $20 \mu \mathrm{g} /$ day dose of ethinyl estradiol. ${ }^{16}$ In this quadriphasic dosing regimen, DNG is increased during week 2 of the cycle while E2V is decreased on day 3 and again on day 25 (Figure 1).

$\mathrm{E} 2 \mathrm{~V}$ is a novel estrogen with structural similarity to $17 \beta$-estradiol and a shorter half-life than ethinyl estradiol. ${ }^{14}$ Therefore, in theory, it may have fewer adverse effects on lipid and glucose metabolism and a decreased risk for thromboembolic or cardiovascular complications. E2V is a prodrug, and upon ingestion is rapidly metabolized to $17 \beta$-estradiol and valeric acid during absorption in the small 


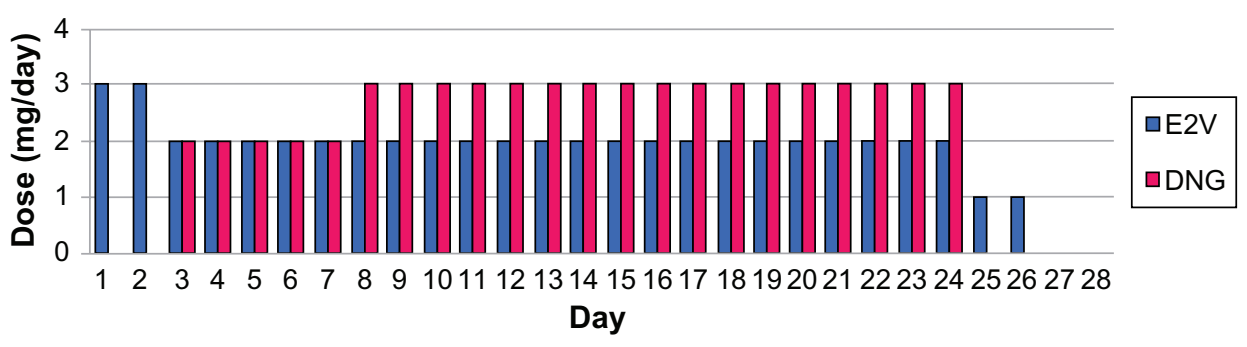

Figure I Daily doses of E2V and DNG in the quadriphasic regimen. Abbreviations: $\mathrm{E} 2 \mathrm{~V}$, estradiol valerate; DNG, dienogest.

intestine and in its first pass through the liver. ${ }^{14} \mathrm{~A} 1 \mathrm{mg}$ dose of $\mathrm{E} 2 \mathrm{~V}$ is equal to $0.76 \mathrm{mg}$ of $17 \beta$-estradiol. ${ }^{16,17}$

$17 \beta$-estradiol is then metabolized to mostly inactive metabolites, ie, estrone, estrone glucuronide, or estrone sulfate, by cytochrome P450 3A enzymes in the liver and intestinal mucosa, resulting in about $5 \%$ of the ingested dose reaching the circulation intact. ${ }^{14,18}$ With this low bioavailability, only $3 \%$ of ingested E2V becomes available as $17 \beta$-estradiol. $17 \beta$-estradiol is highly protein-bound, with $38 \%$ bound to sex hormone binding globulin and $60 \%$ bound to albumin, leaving only $2 \%-3 \%$ unbound. ${ }^{14,18}$ It is excreted mainly in the urine, with only $10 \%$ eliminated in the feces, and has a half-life of approximately 14 hours. ${ }^{14}$

DNG is a unique progestin due to its structure and pharmacologic properties. It is a C-19 nortestosterone derivative, like norethindrone, but is also similar to progesterone derivatives. ${ }^{19}$ Compared with other progestins, DNG has an additional carbon-carbon double bond on its steroidal B ring that results in high activity at progesterone receptors. ${ }^{19}$

DNG is rapidly absorbed, with $90 \%$ bioavailability. ${ }^{14,19}$ It is highly bound (90\%) to albumin, but does not bind to sex hormone binding globulin or corticosteroid binding globulin. ${ }^{14,19}$ About 10\% of DNG circulates unbound, which is a higher percentage than for other nortestosterone derivatives. ${ }^{14,19}$ The lack of DNG binding to sex hormone binding globulin means it does not displace testosterone, resulting in the androgenic effects observed with other progestins..$^{14,19,20}$ Receptor binding studies indicate that it may have antiandrogenic effects similar to cyproterone acetate, a C-21 progestin derivative, but to a lesser degree..$^{20} \mathrm{DNG}$ is metabolized by hydroxylation and conjugation to mostly inactive metabolites that are excreted. ${ }^{14,19}$ Unchanged DNG is the dominant form, and steady state is reached with 2-3 days of once-daily dosing. There is no significant accumulation. ${ }^{14,19}$ The plasma half-life for DNG is about 10 hours, which is similar to that of other nortestosterone derivatives. ${ }^{14,19}$

\section{Efficacy in treating heavy menstrual bleeding}

Studies have specifically evaluated E2V/DNG for its efficacy in heavy and/or prolonged menstrual bleeding. ${ }^{21-23}$ One such multicenter, randomized, double-blind, placebo-controlled Phase III study examined the efficacy and safety of E2V/DNG for the treatment of confirmed heavy menstrual bleeding, prolonged menstrual bleeding, or heavy and prolonged menstrual bleeding. ${ }^{21}$ This study was done in 47 centers across the US and Canada between December 2005 and May 2008, and included a screening phase that lasted up to 28 days, a 90-day run-in interval, a 196-day treatment period, and a 30-day follow-up phase. The study included women 18 years and older who had heavy menstrual bleeding (at least two bleeding episodes with a measured menstrual blood loss $\geq 80 \mathrm{~mL}$ ), prolonged menstrual bleeding (at least two bleeding episodes each lasting $\geq 8$ days), frequent menstrual bleeding (more than five bleeding episodes with a minimum of 20 bleeding days overall), or a combination thereof, and were confirmed by electronic diaries and hemoglobin extraction from collected sanitary protection for quantification of blood loss. Participants had to use a barrier method of contraception, use only sanitary protection items (pads and tampons) provided to them, and have a normal endometrial biopsy or, at most, mild simple endometrial hyperplasia during the six months before study entry. Women older than 40 years had to have a follicle-stimulating hormone level less than $40 \mathrm{mIU} / \mathrm{mL}$. Women were excluded if they had an abnormal transvaginal ultrasonogram, any clinically significant abnormal laboratory value, endometrial ablation or dilatation and curettage within two months of the study, or organic pathology (eg, von Willebrand disease, chronic endometritis, adenomyosis, endometriosis) for their heavy and prolonged menstrual bleeding. Women were also excluded if they used symptomatic treatment for AUB (eg, NSAIDs), had a body mass index $>32$, smoked $>10$ cigarettes per day (if aged $>35$ years), or met 
other criteria consistent with contraindications for the use of combined oral contraceptives. Iron supplementation was allowed if the attending physician deemed it necessary.

Women who met the inclusion criteria with at least one qualifying symptom were randomized (2:1) to E2V/DNG or matching placebo for 196 days (seven cycles). Data from the last 90 days and initial (run-in phase) 90 days were compared. The primary outcome was the proportion of women with a complete response to treatment (ie, a return to complete menstrual normality) defined as achieving all qualifying conditions, ie, no bleeding episodes that lasted more than seven days, not more than four bleeding episodes, no bleeding episodes that involved blood loss $\geq 80 \mathrm{~mL}$, no more than one bleeding episode increase from baseline, no more than 24 days of bleeding, and no increase from baseline for an individual's total number of bleeding days. Participants were categorized as complete, partial, or nonresponders (missed at least one of the relevant criteria for a complete response), or participants with missing data.

Data from 120 women randomized to E2V/DNG and 70 women to placebo were evaluated. The proportion of complete responders in the intention-to-treat analysis was significantly higher in the E2V/DNG group compared with the placebo group $(29.2 \%$ and $2.9 \%$, respectively; $P<0.001$ ). For participants with evaluable data (ie, excluding those with missing data), a complete response was seen in $43.8 \%(35 / 80)$ of women taking E2V/DNG and $4.2 \%(2 / 48)$ of women taking placebo $(P<0.001)$. The proportion of patients experiencing resolution of heavy bleeding (ie, no episodes with $\geq 80 \mathrm{~mL}$ menstrual blood loss) was much higher in the treatment group (56.0\%) than in the placebo group (26.7\%). An $80 \%$ reduction in menstrual blood loss was found for $45 \%$ of women in the E2V/DNG group and 5\% in the placebo group. The mean adjusted between-treatment difference for menstrual blood loss was a $252 \mathrm{~mL}$ reduction $(95 \% \mathrm{CI}-339$ to $-165 ; P<0.001)$ and the mean adjusted between-treatment difference over 90 days for sanitary protection items used was 23 fewer items (95\% CI -39 to $-8 ; P<0.001$ ), favoring E2V/DNG. Statistically significant improvements were also seen in various measures of iron metabolism (ie, ferritin, hemoglobin, and hematocrit).

This study demonstrated the efficacy of E2V/DNG in women with heavy and prolonged menstrual bleeding without organic pathology. Strict inclusion criteria and difficult logistic requirements for adherence (eg, collecting all sanitary products used) may have limited recruitment. Further, strict criteria were applied for participants to be considered a complete responder which may not be a realistic clinical measure.

Another study with very similar methodology was performed at 34 centers in Europe and Australia from February 2006 through May 2008. ${ }^{22}$ Similar to the previous study, the primary efficacy outcome was the proportion of women who showed a complete response to treatment. The intention-to-treat population comprised of 231 women (149 randomized to E2V/DNG and 82 randomized to placebo). The proportion of complete responders was significantly higher in both the intention-to-treat and evaluable data populations. In the intention-to-treat population, $29.5 \%$ of women receiving E2V/DNG versus $1.2 \%$ of those receiving placebo had a complete response $(P<0.0001)$. In women with an evaluable response (ie, those with no missing data), $40.7 \%$ receiving E2V/DNG versus $1.6 \%$ of those receiving placebo had a complete response $(P<0.0001)$. More than $90 \%$ of women recruited had heavy menstrual bleeding; $63.2 \%$ of women treated with E2V/DNG responded with $<80 \mathrm{~mL}$ menstrual blood loss during each episode compared with $14.5 \%$ of women taking placebo. Secondary findings included an $80 \%$ reduction in menstrual blood loss for $50 \%$ of women in the E2V/DNG group and $0 \%$ in the placebo group. The mean adjusted betweentreatment difference for menstrual blood loss was $373 \mathrm{~mL}$ in favor of E2V/DNG $(95 \%$ CI $255-490 ; P<0.0001)$ and the mean adjusted between-treatment difference over 90 days for sanitary protection used was 22 fewer items (95\% CI -30 to $-14 ; P<0.0001)$. Marked improvements were also seen in various measures of iron metabolism (ie, ferritin, hemoglobin, and hematocrit). Investigators and patients alike noted significantly improved bleeding symptoms with E2V/ DNG compared with placebo. Given the limitations, which were similar to those of the previous study, this study also demonstrated effectiveness for E2V/DNG in women with heavy and prolonged menstrual bleeding.

In a pooled analysis of the two aforementioned studies, the efficacy of E2V/DNG in the treatment of heavy and prolonged menstrual bleeding without organic pathology was evaluated. ${ }^{23}$ Women 18 years or older were randomized to E2V/DNG $(n=269)$ or placebo $(n=152)$ for 196 days. The efficacy endpoints in this study were changes in menstrual blood loss, number of sanitary protection items used, and iron metabolism parameters.

In the intention-to-treat population, the reduction in menstrual blood loss with E2V/DNG was rapid and sustained over the duration of treatment for seven cycles. After six months of treatment, median menstrual blood 
loss was reduced by $88 \%$ compared with a $24 \%$ reduction with placebo. The mean absolute total reduction in 90-day menstrual blood loss from baseline to the 90-day efficacy phase was $414 \pm 373 \mathrm{~mL}$ with E2V/DNG and $109 \pm 300 \mathrm{~mL}$ with placebo $(P<0.0001)$. Overall, an $80 \%$ reduction in menstrual blood loss was achieved by $46 \%$ of women in the $\mathrm{E} 2 \mathrm{~V} / \mathrm{DNG}$ group and $2 \%$ of women in the placebo group. The mean between-treatment difference in amount of sanitary protection used significantly favored $\mathrm{E} 2 \mathrm{~V} / \mathrm{DNG}$, with 22.1 fewer items $(95 \% \mathrm{CI}-30.7$ to $-13.6 ; P<0.0001)$. Markedly significant improvements in hemoglobin, hematocrit, and ferritin were observed in women receiving E2V/DNG compared with little or no improvement in women receiving placebo. Specifically, the change from baseline for hemoglobin $(\mathrm{g} / \mathrm{dL})$ was $0.64 \pm 1.1$ with E2V/ DNG compared with $0.12 \pm 1.0$ for placebo $(P<0.0001)$; the change from baseline for hematocrit (\%) was $1.48 \pm$ 3.7 with E2V/DNG compared with $0.08 \pm 3.1$ for placebo $(P=0.0002)$; and the change from baseline for ferritin (ng/ $\mathrm{mL})$ was $7.1 \pm 28.8$ compared with $1.2 \pm 12.2$ for placebo $(P<0.05)$.

This pooled analysis was recently reanalyzed using a definition of treatment success (used in other studies) as menstrual blood loss less than $80 \mathrm{~mL}$ together with a reduction in menstrual blood loss of $\geq 50 \%$ from baseline. ${ }^{24}$ This analysis was restricted to those subjects with heavy menstrual bleeding and included 220 women randomized to E2V/ DNG and 135 women randomized to placebo. At study end (cycle 7 or last observation carried forward), a significantly greater proportion of women achieved successful treatment with E2V/DNG (63.6\%) compared with placebo (11.9\%), with a corresponding difference of $51.8 \%$ (95\% CI 43.4-60.2; $P<0.001)$. The proportion of women with menstrual blood loss less than $80 \mathrm{~mL}$ was $68.2 \%$ using E2V/DNG compared with $15.6 \%$ using placebo (corresponding difference 52.6\%; 95\% CI 44.0-61.3; $P<0.001)$ and the proportion of women with a menstrual blood loss reduction $\geq 50 \%$ from baseline was $70.0 \%$ for those using E2V/DNG and $17 \%$ for those using placebo (corresponding differences 53.0\%; 95\% CI 44.2-61.7; $P<0.001)$.

These pooled analyses demonstrated that E2V/DNG rapidly reduced menstrual blood loss in women with heavy and prolonged menstrual bleeding upon treatment initiation and over treatment duration. These pooled analyses show that the decrease in menstrual blood loss is consistent across a larger and more diverse population. This decrease in menstrual blood loss was associated with use of fewer sanitary protection items and improved iron metabolism parameters. It should be noted that E2V/DNG has not yet been compared with other forms of contraception in women with heavy menstrual bleeding (eg, other combination oral contraceptives, LNG-IUD). E2V/DNG has shown significantly improved bleeding patterns compared with ethinyl estradiol/LNG in healthy women aged 18-50 years without heavy menstrual bleeding. ${ }^{25}$ While effective for contraception, E2V/DNG has the added advantage of reducing menstrual blood loss and should be considered when assessing treatment options for patients with heavy and prolonged menstrual bleeding without organic pathology.

\section{Safety}

The safety profile of E2V/DNG is similar to that of other combination oral contraceptives containing $35 \mu \mathrm{g}$ or less of ethinyl estradiol. With the theoretically lower adverse effect profile of E2V with regard to lipid and glucose metabolism, there may be a decrease in the rate and severity of thromboembolic complications. There is a paucity of comparative data to prove or disprove these effects. One open-label trial comparing E2V/DNG with ethinyl estradiol/LNG showed slight improvement of high-density lipoprotein in the E2V/DNG group. However, there was no significant difference in low-density lipoprotein, carbohydrate metabolism, or hemostasis between the two groups. ${ }^{26}$ Other studies showed that E2V/DNG has no significant effect on coagulation factors when compared with ethinyl estradiol/ LNG. This includes factor VII, VIII, antithrombin III, proteins $\mathrm{C}$ and $\mathrm{S}$, or inactivated protein $\mathrm{C} .{ }^{27} \mathrm{E} 2 \mathrm{~V} / \mathrm{DNG}$ also had no effect on D-dimer or fibrinogen levels when compared with ethinyl estradiol/LNG. ${ }^{28}$ Currently only one case each of myocardial infarction and deep vein thrombosis have been reported in the literature for patients taking E2V/DNG. ${ }^{14}$ Studies currently ongoing are appropriately powered to show whether E2V/DNG has an increased or decreased risk of thromboembolic and cardiovascular events. ${ }^{29}$ Until the results of these studies are available, clinicians should apply the same precautions and contraindications for use of combination oral contraceptives containing ethinyl estradiol to use of E2V.

Common side effects of $\mathrm{E} 2 \mathrm{~V} / \mathrm{DNG}$ are similar to those found with other combination oral contraceptives. In a combined report of three studies consisting of over 2200 patients, the most common adverse events were breast discomfort (4.9\%), dysmenorrhea (4.9\%), headache $(3.1 \%)$, and acne $(2.8 \%) .{ }^{30}$ Other common adverse events reported included increased weight, amenorrhea, and abdominal pain. The most common reasons for discontinuing E2V/DNG were breakthrough bleeding (metrorrhagia), acne, and weight 
gain. ${ }^{17}$ In a study comparing E2V/DNG and ethinyl estradiol/ LNG, adverse effects were similar between the two groups. ${ }^{25}$ The most commonly reported side effects in the E2V/DNG group were breast pain, headache, and vaginal infections compared with acne, headache, and nasopharyngitis in the ethinyl estradiol/LNG group. There was no statistically significant difference in the number of adverse events reported for the two groups.

Serious adverse events that could be possibly linked to treatment with E2V/DNG were rare, and included presumed ocular histoplasmosis syndrome, uterine leiomyoma, focal nodular hyperplasia of the liver, myocardial infarction, deep vein thrombosis, ruptured ovarian cyst, autonomic nervous system imbalance, and breast cancer. There was only one reported case per event and other causes could not be ruled out. ${ }^{31}$

At this time, no distinct safety advantages or disadvantages can be considered when comparing E2V/DNG with other combination oral contraceptives containing ethinyl estradiol $35 \mu \mathrm{g}$ or less. More studies are needed to characterize further the impact on lipid and glucose metabolism, and ultimately determine if there is less risk of thromboembolic or cardiovascular events.

\section{Place in therapy}

Several options exist for the treatment of heavy menstrual bleeding. The best option for a patient depends on etiology, severity of bleeding (interference with daily activities), associated symptoms (ie, pelvic pain), contraindications to hormonal or other medications, contraceptive needs, future pregnancy plans, comorbidities, and patient preference for length of treatment. Finally, treatment cost must be considered. Treatment options may include surgery or medications, such as NSAIDs, antifibrinolytics (ie, tranexamic acid), or hormonal modulators (ie, danazol), including oral progestins, combination oral contraceptives, gonadotropin receptor antagonists, and LNG-IUD. Only primary and secondary medical treatment options for chronic heavy menstrual bleeding will be discussed. E2V/DNG will be compared with other treatment options for heavy menstrual bleeding. Antifibrinolytics and hormonal modulators are not preferred agents and are not discussed here.

\section{Heavy menstrual bleeding in sexually active women with anovulatory or ovulatory cycles}

The American College of Obstetrics and Gynecology recommends that patients with anovulatory or ovulatory bleeding be treated with a combination oral contraceptive if they require contraception..$^{32,33}$ The LNG-IUD has shown superior efficacy to combination oral contraceptives and for this reason may be considered first-line treatment. ${ }^{34,35}$ LNG-IUD reduces heavy menstrual bleeding by $95 \%$ after three months. ${ }^{36} \mathrm{E} 2 \mathrm{~V} / \mathrm{DNG}$ is an appropriate first choice for most premenopausal women who have a need for contraception, do not have contraindications to estrogen therapy, and do not desire insertion of a LNG-IUD. No comparative trials of E2V/DNG versus LNG-IUD have been conducted; until these studies are completed, the LNG-IUD is considered better than E2V/DNG given the superiority of the LNG-IUD data compared with those for other combination oral contraceptives used to treat long-term heavy menstrual bleeding. ${ }^{34,45}$

As discussed earlier, E2V/DNG was more effective than placebo in patients with heavy menstrual bleeding in reducing bleeding volume and has been given an indication for this condition..$^{21,22}$ These trials were six months in duration, and long-term comparative trials versus LNG-IUD are needed. To compare combination oral contraceptives in healthy women seeking contraception, one study performed in 798 women aged 18-50 years demonstrated improved bleeding patterns with seven cycles of E2V/DNG $(n=399)$ compared with a combination oral contraceptive containing ethinyl estradiol/ LNG $(\mathrm{n}=399) .{ }^{25}$ There were significantly fewer bleeding/ spotting days reported by women receiving E2V/DNG compared with ethinyl estradiol/LNG $(17.3 \pm 10.4$ versus $21.5 \pm 8.6$, respectively, $P<0.0001)$ and better cycle control (more cycles with absent withdrawal bleeding in the E2V/ DNG group, $P<0.001)$. Scheduled withdrawal bleeding episodes were shorter (median 4.0 versus 5.0 days, $P<0.05$ ) and lighter in women treated with E2V/DNG versus ethinyl estradiol/LNG. While this study was not performed in women specifically with heavy menstrual bleeding, it provides evidence that E2V/DNG may be a good oral contraceptive option for women with menstrual disturbances.

Premenopausal ovulatory patients not desiring pregnancy within the upcoming year who have contraindications to estrogen therapy may benefit most from LNG-IUD. Patients not planning a pregnancy who are anovulatory may benefit from E2V/DNG as a first-line option. Alternatively, a cyclic oral progestin or LNG-IUD could be used. ${ }^{32}$

\section{Heavy menstrual bleeding in nonsexually active women with anovulatory or ovulatory cycles}

Anovulatory bleeding in women who are not sexually active may be treated with an oral contraceptive, cyclic progestin, or an NSAID. ${ }^{32} \mathrm{~A}$ combination oral contraceptive 
such as E2V/DNG is an appropriate option for women experiencing ovulatory or anovulatory cycles, given the data from studies mentioned above. A progestin such as micronized progesterone or medroxyprogesterone acetate given cyclically (for 14-16 days of the month prior to the anticipated start of the menstrual cycle) or the LNG-IUD are also first-line options.

Cost and daily compliance may be considered in patients who are not sexually active. A significant difference in treatment costs exists (Table 1). Cost differences may limit treatment with $\mathrm{E} 2 \mathrm{~V} / \mathrm{DNG}$, other combination oral contraceptive, or progestin if not covered by insurance. An NSAID may be the best treatment option for those with ovulatory heavy menstrual bleeding, who do not want surgery, cannot receive hormone therapy, or have insurance difficulties. Ibuprofen and meclofenamic acid are commonly used, but no single NSAID has shown superior efficacy. ${ }^{37,38}$ NSAID treatments typically last 5 days or until menses ends. NSAIDS reduce blood loss by $20 \%-40 \%$ and much greater reductions have been shown in patients with excessive bleeding. ${ }^{39,40}$ Patients using E2V/DNG average around a $65 \%$ reduction in mean blood loss while nearly half experience up to an $80 \%$ reduction in mean blood loss. ${ }^{21}$ Thus, E2V/DNG may be cost-effective compared with an NSAID if it successfully controls heavy menstrual bleeding. Patients with compliance issues may benefit most from the LNG-IUD.

\section{Heavy menstrual bleeding in women who plan to conceive in the upcoming year}

$\mathrm{E} 2 \mathrm{~V} / \mathrm{DNG}$ is not an appropriate choice for patients with ovulatory cycles who are attempting conception within a year. These women may want to avoid E2V/DNG, along with other combination oral contraceptives, and instead use

Table I Cost comparison of treatments for heavy menstrual bleeding ${ }^{36}$

\begin{tabular}{ll}
\hline Drug & $\begin{array}{l}\text { Annual Treatment Cost, } \\
\text { Estimated (United States } \\
\text { dollars) }\end{array}$ \\
\hline Estradiol valerate/dienogest & $\$ 1 \mid 20-\$ 1136$ \\
Levonorgestrel IUD* & $\$ 844$ \\
lbuprofen** & $\$ 22$ \\
Ethinyl estradiol/levonorgestrel & $\$ 358-\$ 374$ \\
Micronized progesterone*** & $\$ 1454-\$ 1488$ \\
Medroxyprogesterone acetate**** & $\$ 90-\$ 180$ \\
\hline
\end{tabular}

Notes: *One time cost does not reflect procedural service for insertion; **assumes daily dose of ibuprofen $600 \mathrm{mg}$ daily $\times 5$ days, so 5 tablets; ***assumes daily dose of $300 \mathrm{mg}$; ****assumes daily dose of $10 \mathrm{mg}$ although for very heavy bleeding $10 \mathrm{mg}$ twice daily is needed.

Abbreviation: IUD, intrauterine device. an NSAID or an oral cyclic progestin, such as micronized progestin or medroxyprogesterone acetate. ${ }^{32}$

\section{Heavy menstrual bleeding in women with a concomitant condition}

Patients with fibroids and heavy menstrual bleeding are best treated with gonadotropin-releasing hormone agonists or NSAIDs if drug therapy is chosen over surgical treatment. ${ }^{38,41,42}$ Although E2V/DNG has not been studied in these patients, other combination oral contraceptives have been used successfully to decrease the volume and duration of blood loss to the levels seen in patients without fibroids. Thus, E2V/DNG may be an option for these patients.

The US Medical Eligibility Criteria for Contraceptive Use or the World Health Organization Medical Eligibility Criteria for Contraceptive Use can be used to determine whether women with concurrent conditions are candidates for heavy menstrual bleeding therapies that also function as contraceptives. ${ }^{43,44}$ Women with heavy menstrual bleeding and a high risk of cardiovascular disease require nonestrogen-based treatment. ${ }^{43}$ Risks include older age, smoking, hypertension, recent major surgery, history of arterial or venous thrombosis, or medical conditions, such as inherited thrombophilia, active cancer, or diabetes. ${ }^{43}$ E2V/DNG should be used with caution in obese patients since the safety and efficacy have not been adequately evaluated in women with a body mass index (BMI) $>30 \mathrm{~kg} / \mathrm{m}^{2}$ (average BMI 24.6 to $26 \mathrm{~kg} / \mathrm{m}^{2}$ among study participants, up to $\left.32 \mathrm{~kg} / \mathrm{m}^{2}\right) .{ }^{14} \mathrm{LNG}-\mathrm{IUD}$, cyclic progestin, and NSAID are alternative options for women with heavy menstrual bleeding and a concurrent condition. ${ }^{32,33}$

\section{Duration of therapy preferences}

Patients must be informed of the efficacy of the different treatment options for heavy menstrual bleeding. Given these options, clinicians need to provide guidance according to the length of treatment desired by the patient. Daily, short-term, or long-term options are available. Patients who do not want a daily oral regimen and are not planning to conceive in the next year may be better suited for insertion of an LNG-IUD. Patients without contraindications who prefer a daily regimen may benefit most from E2V/DNG or other combination oral contraceptives. Those who prefer a monthly two-week course may be best suited to cyclic progestin. Patients who desire a very short course (five days or less) may consider an NSAID.

\section{Conclusion}

$\mathrm{E} 2 \mathrm{~V} / \mathrm{DNG}$ is a novel combination oral contraceptive recently approved for heavy menstrual bleeding. E2V/DNG has been 
found to be more efficacious than placebo in reducing heavy menstrual bleeding and improving hematologic indices. In healthy women without heavy menstrual bleeding, E2V/ DNG was found to decrease the bleeding duration and induce amenorrhea more effectively than ethinyl estradiol/ LNG. E2V/DNG has a safety profile similar to that of other combination oral contraceptives. This product is best suited to women with heavy menstrual bleeding who are anovulatory or ovulatory and require contraception. LNG-IUD may be the most appropriate choice in patients not desiring conception for at least one year or who do not want a daily or cyclic pill burden. NSAIDS are an option for ovulating patients who are not sexually active but require a cost-effective or short-course treatment. Cyclic progestins may be best suited to anovulatory or ovulatory patients with contraindications to estrogen who plan for pregnancy within a year. When compared with alternative treatment options, E2V/DNG has the added benefit of providing effective contraception. Further studies are needed to compare the efficacy of E2V/LNG with that of other combination oral contraceptives, the LNG-IUD, and other standard therapies in women with heavy menstrual bleeding.

\section{Disclosure}

The authors have no conflicts of interest to report in this work.

\section{References}

1. Cote I, Jacobs P, Cumming DC. Use of health services associated with increased menstrual loss in the United States. Am J Obstet Gynecol. 2003;188:343-348.

2. Spencer CP, Whitehead MI. Endometrial assessment re-visited. $\mathrm{Br} J$ Obstet Gynaecol. 1999;106:623-632.

3. Thompson BL, Ponce de Leon R, Kieke B, Velebil P, Wingo PA. Trends in hospitalizations for abnormal uterine bleeding in the United States: 1980-1992. J Women's Health. 1997;6:73-81.

4. Shapley M, Jordan K, Croft PR. Increased vaginal bleeding and psychological distress: a longitudinal study of their relationship in the community. BJOG. 2003;110:548-554.

5. Cote I, Jacobs P, Cumming D. Work loss associated with increased menstrual loss in the United States. Obstet Gynecol. 2002;100: 683-687.

6. Munro MG, Critchley HO, Broder MS, Fraser IS. FIGO classification system (PALM-COEIN) for causes of abnormal uterine bleeding in nongravid women of reproductive age. Int $J$ Gynaecol Obstet. 2011;113:3-13.

7. Liu Z, Doan QV, Blumenthal P, Dubois RW. A systematic review evaluating health-related quality of life, work impairment, and healthcare costs and utilization in abnormal uterine bleeding. Value Health. 2007;10:183-194.

8. American College of Obstetricians and Gynecologists. Practice bulletin no 128: diagnosis of abnormal uterine bleeding in reproductive-aged women. Obstet Gynecol. 2012;120:197-206.

9. Fraser IS, Critchley HO, Munro HG, Broder M. A process designed to lead to international agreement on terminologies and definitions to be used to describe abnormalities of menstrual bleeding. Fertil Steril. 2007;87:466-476.
10. James AH. More than menorrhagia: a review of the obstetric and gynaecological manifestations of bleeding disorders. Haemophilia. 2005;11:295-307.

11. Shankar M, Lee CA, Sabin CA, Economides DL, Kadir RA. von Willebrand disease in women with menorrhagia: a systematic review. BJOG. 2004:734-740.

12. Dilley A, Drews C, Miller C, et al. von Willebrand disease and other inherited bleeding disorders in women with diagnosed menorrhagia. Obstet Gynecol. 2001;97:630-636.

13. Kadir RA, Economides DL, Sabin CA, Owens D, Lee CA. Frequency of inherited bleeding disorders in women with menorrhagia. Lancet. 1998;351:485-489.

14. Natazia (estradiol valerate/dienogest) [package insert]. Wayne, NJ: Bayer HealthCare Pharmaceuticals; 2012. Available from: http://labeling. bayerhealthcare.com/html/products/pi/natazia_pi.pdf.AccessedApril9, 2013.

15. Fruzzetti F, Bitzer J. Review of clinical experience with estradiol in combined oral contraceptives. Contraception. 2010;81:8-15.

16. Kiley JW, Shulman LP. Estradiol valerate and dienogest: a new approach to oral contraception. Int J Womens Health. 2011;3:281-286.

17. Palacios S, Wildt L, Parke S, Machlitt A, Römer T, Bitzer J. Efficacy and safety of a novel oral contraceptive based on oestradiol (oestradiol valerate/dienogest): a Phase III trial. Eur J Obstet Gynecol Reprod Biol. 2010;149:57-62.

18. Zeun S, Lu M, Uddin A, et al. Pharmacokinetics of an oral contraceptive containing oestradiol valerate and dienogest. Eur J Contracept Reprod Health Care. 2009;14:221-232.

19. Ruan X, Seeger H, Mueck AO. The pharmacology of dienogest. Maturitas. 2012;71:337-344.

20. Sasagawa S, Shimizu Y, Kami H, et al. Dienogest is a selective progesterone receptor agonist in transactivation analysis with potent oral endometrial activity due to its efficient pharmacokinetic profile. Steroids. 2008;73:222-231.

21. Jensen JT, Parke S, Mellinger U, MachlittA, Fraser IS. Effective treatment of heavy menstrual bleeding with estradiol valerate and dienogest: a randomized controlled trial. Obstet Gynecol. 2011;117:777-787.

22. Fraser IS, Romer T, Parke S, et al. Effective treatment of heavy and/or prolonged menstrual bleeding with an oral contraceptive containing estradiol valerate and dienogest: a randomized, double-blind Phase III trial. Hum Reprod. 2011;26:2698-2708.

23. Fraser IS, Parke S, Mellinger U, Machlitt A, Serrani M, Jensen J. Effective treatment of heavy and/or prolonged menstrual bleeding without organic cause: pooled analysis of two multinational, randomized, double-blind, placebo-controlled trials of oestradiol valerate and dienogest. Eur $J$ Contracept Reprod Health Care. 2011;16:258-269.

24. Fraser IS, Jensen J, Schaefers M, Mellinger U, Parke S, Serrani M. Normalization of blood loss in women with heavy menstrual bleeding treated with an oral contraceptive containing estradiol valerate/ dienogest. Contraception. 2012;86:96-101.

25. Ahrendt HJ, Makalova D, Parke S, Mellinger U, Mansour D. Bleeding pattern and cycle control with an estradiol-based oral contraceptive: a seven cycle, randomized, comparative trial of estradiol valerate/ dienogest and ethinyl estradiol/levonorgestrel. Contraception. 2009;80: 436-444.

26. Parke S, Nahum G, Mellinger U, Junge W. Metabolic effect of a new four-phasic oral contraceptive containing estradiol valerate and dienogest. Obstet Gynecol. 2008;111 Suppl 4:S12-S13.

27. Hoy SM, Scott LJ. Estradiol valerate/dienogest: in oral contraception. Drugs. 2009;69:1635-1646.

28. Parke S, Junge W, Mellinger U, Dujkers I, Klipping C. Comparative effects of a four-phasic regimen of estradiol valerate/dienogest versus ethinyl estradiol/levonorgestrel on haemostatic parameters. Hum Reprod. 2008;23 Supp1 1:i78-i79.

29. International Active Surveillance Study-Safety of Contraceptives: Role of Estrogens (INAS-SCORE). Available from: from: http://clinicaltrials. gov/. Accessed January 31, 2013. 
30. Nelson A, Sampson-Landers C, Parke S, Jensen JT. Efficacy of estradiol valerate/dienogest OC: results of 3 large studies in North America and Europe. Abstract presented at the 57th Annual Clinical Meeting of the American Congress of Obstetricians and Gynecologists, May 2-6, 2009, Chicago, IL.

31. Borgelt LM, Martell CW. Estradiol valerate/dienogest: a novel combined oral contraceptive. Clin Ther. 2012;34:37-55.

32. American College of Obstetricians and Gynecologists. ACOG practice bulletin: management of anovulatory bleeding. Int J Gynaecol Obstet. 2001;72:263-271.

33. [No authors listed]. ACOG practice bulletin: Noncontraceptive uses of hormonal contraceptives. Obstet Gynecol. 2010;110:206-218.

34. Shaaban MM, Zakherah MS, El-Nashar SA, et al. Levonorgestrelreleasing intrauterine system compared to low dose combined oral contraceptive pills for idiopathic menorrhagia: a randomized clinical trial. Contraception. 2011;83:48-54.

35. Gupta J, Kai J, Middleton L, Pattison H, Gray R, Daniels J. Levonorgestrel intrauterine system versus medical therapy for menorrhagia. $N$ Engl J Med. 2013;368:128-137.

36. Lethaby AE, Cooke I, Rees M. Progesterone or progestogen-releasing intrauterine systems for heavy menstrual bleeding. Cochrane Database Syst Rev. 2005;19:CD002126.

37. Fraser IS, McCarron G. Randomized trial of 2 hormonal and 2 prostaglandin-inhibiting agents in women with a complaint of menorrhagia. Aust N Z J Obstet Gynaecol. 1991;31:66-70.
38. Mäkäräinen L, Ylikorkala O. Primary and myoma-associated menorrhagia: role of prostaglandins and effects of ibuprofen. $\mathrm{Br} J$ Obstet Gynaecol. 1986;93:974-978.

39. Hall P, Maclachlan N, Thorn N, Nudd MW, Taylor CG, Garrioch DB. Control of menorrhagia by the cyclo-oxygenase inhibitors naproxen sodium and mefenamic acid. Br J Obstet Gynaecol. 1987;94:554-558.

40. Lethaby A, Augood C, Duckitt K. Nonsteroidal anti-inflammatory drugs for heavy menstrual bleeding. Cochrane Database Syst Rev. 2002;1: CD000400.

41. Carr BR, Marshburn PB, Weatherall PT, et al. An evaluation of the effect of gonadotropin-releasing hormone analogs and medroxyprogesterone acetate on uterine leiomyomata volume by magnetic resonance imaging: a prospective, randomized, double blind, placebo-controlled, crossover trial. J Clin Endocrinol Metab. 1993;76:1217-1223.

42. Marshall LM, Spiegelman D, Goldman MB, et al. A prospective study of reproductive factors and oral contraceptive use in relation to the risk of uterine leiomyomata. Fertil Steril. 1998;70:432-439.

43. Centers for Disease Control and Prevention. US medical eligibility criteria for contraceptive use, 2010. MMWR. Available from: http:// www.cdc.gov/mmwr/pdf/rr/rr59e0528.pdf. Accessed April 9, 2013.

44. World Health Organization. Medical Eligibility Criteria for Contraceptive Use, 4th ed. Geneva, Switzerland: World Health Organization; 2009. Available from: http://www.who.int/reproductivehealth/publications/ family_planning/9789241563888/en/index.html. Accessed April 9, 2013.
International Journal of Women's Health

\section{Publish your work in this journal}

The International Journal of Women's Health is an international, peerreviewed open-access journal publishing original research, reports, editorials, reviews and commentaries on all aspects of women's healthcare including gynecology, obstetrics, and breast cancer. The manuscript management system is completely online and includes

\section{Dovepress}

a very quick and fair peer-review system, which is all easy to use. Visit http://www.dovepress.com/testimonials.php to read real quotes from published authors. 